\title{
Genetic Evidence for Reproductive Isolation Among Sympatric Epichloë Endophytes as Inferred from Newly Developed Microsatellite Markers
}

\author{
Melanie K. Schirrmann • Stefan Zoller • Simone Fior • \\ Adrian Leuchtmann
}

Received: 3 June 2014 / Accepted: 9 December 2014 /Published online: 28 December 2014

(C) Springer Science+Business Media New York 2014

\begin{abstract}
Reproductive isolation is central to the maintenance of species, and especially in sympatry, effective barriers to prevent interspecific crosses are expected. Host specificity is thought to constitute an effective mechanism for the formation of barriers in different genera of Fungi, but evidence for endophytes is so far lacking. Sexual Epichloë species (Ascomycota, Clavicipitaceae) represent an ideal study system to investigate the mechanisms underlying speciation as mediated by host specificity because they include species complexes with several host-specific taxa. Here, we studied genetic differentiation of three host-specific Epichloë species using microsatellite markers that were newly in silico identified on the genome of Epichloë poae. Among these, 15 were experimentally tested and applied to study an extensive sampling of isolates representing Epichloë typhina infecting Dactylis glomerata and Epichloë clarkii infecting Holcus lanatus from a site with sympatric populations in Switzerland, as well as a reduced sampling of E. poae infecting Poa nemoralis to create a three-taxon dataset. Both principal coordinate analysis and Bayesian clustering algorithm showed three genetically distinct groups representing the three host-specific species. High pairwise $F_{\mathrm{ST}}$ values among the three species, as well as sequencing data of the tefA gene revealing diagnostic single nucleotide polymorphisms (SNPs), further support the hypothesis of genetic
\end{abstract}

Electronic supplementary material The online version of this article (doi:10.1007/s00248-014-0556-5) contains supplementary material, which is available to authorized users.

M. K. Schirrmann $(\bowtie) \cdot$ S. Fior $\cdot$ A. Leuchtmann ETH Zurich, Institute of Integrative Biology (IBZ), Universitätstrasse 16, 8092 Zürich, Switzerland

e-mail: melanie.schirrmann@env.ethz.ch

S. Zoller

ETH Zurich, Genetic Diversity Centre (GDC), Universitätstrasse 16, 8092 Zürich, Switzerland discontinuities among the taxa. These results provide genotypic evidence of the maintenance of reproductive isolation of the species in a context of sympatry. In silico testing of 885 discovered microsatellites on the genome of Epichloëfestucae extend their applicability to a wider taxonomic range of Epichloë.

Keywords Endophytes $\cdot$ Microsatellites $\cdot$ Population structure $\cdot$ Reproductive isolation $\cdot$ Sympatry

\section{Introduction}

Speciation in fungi can occur through numerous mechanisms resulting in the formation of isolating barriers among divergent lineages. Compared to speciation in plants and animals, where geographic separation has long been hypothesized to be a strong driver in the formation of current biodiversity, the mechanisms underlying speciation in fungi have been less amenable to a general consensus [1-3]. Indeed, as eukaryotic microorganisms typically have global geographic ranges, geographic isolation has been questioned as a promoter of divergence [4]. Similarly, the process of sympatric speciation has often been regarded as unlikely because minimal gene flow is sufficient to prevent differentiation [5]. However, recent advances facilitated by the use of genome-wide data in wellestablished model systems $[6,7]$ are greatly contributing to the understanding of speciation in fungi, and evidence is emerging for both types of speciation processes. Several instances have been described, where divergence originated in a context of allopatry, but speciation remained cryptic because of undistinguishable phenotypes within species complexes [8-10]. On the other hand, speciation in sympatry may indeed 
occur following the formation of isolating barriers, and in this context, adaptation to different hosts has been proven to be a strong driver of this process [11-13]. Because of the difficulty to separate between development and mating, mutations providing adaptation to a new habitat can pleiotropically affect both the fitness and the ability to mate in this habitat, which can thus be sufficient to restrict gene flow in sympatry $[2,14]$. However, whether host specificity is indicative of different species in a context of sympatry depends on the efficiency of the isolating barriers that prevent admixtures of genotypes, which should be proven with population genetic data.

Sexual species of Epichlö (Ascomycota, Clavicipitaceae) constitute an ideal study system to investigate the genetics behind reproductive isolation, as they form host-adapted species complexes with pooid grasses (Poaceae) that are maintained in sympatric populations under a strong depletion of natural hybrids [15-17]. The endophytes grow in plant apoplasts primarily in shoot apical meristems and leaf sheaths [18] with little or no negative effects on the vegetative tissues of their hosts. However, during their sexual life cycle, they may form external reproductive structures, i.e., stromata, that partially or completely inhibit host flowering and seed production. The effect of such interaction on the plant is known as "choke disease" [19]. Sexual species of Epichloë are heterothallic and possess a bipolar self-incompatibility mating system [20]. Successful mating, therefore, requires transfer of spermatia (male gametes) between stromata of opposite mating types, usually mediated by specialized flies of the genus Botanophila (Anthomyiidae) in a process similar to pollination [21].

Most sexual Epichloë species are circumscribed based on their reproductive incompatibilities with other species following a biological species concept [22]. Based on intersterility tests between species, nine different mating populations corresponding usually to a single taxonomic Epichloë species were identified [23]. Each species typically shows a rather narrow host range restricted to grass species of one genus or closely related host genera, with the exception of Epichlö typhina that forms a complex with several host-specific taxa [22]. Some of the species of the E. typhina complex have been demonstrated to be able to hybridize in artificial experiments of cross-fertilization, but appear to remain reproductively isolated in natural populations [24, 25].

A particular case where this is evident to a large extent includes Epichlö̈ clarkii infecting Holcus lanatus and E. typhina infecting Dactylis glomerata. These host-specific species are morphologically well defined [26] and occur scattered at multiple locations of their distribution range throughout temperate Europe and western Asia. In a recent field study, Bultman et al. [27] described sympatric populations of the two species infecting hosts growing intermixed at the site of Aubonne in Canton Vaud, Switzerland. Here, hybrid ascospores were reported in a small fraction ( 9 \%) of the fertilized stromata; however, capability of these spores to infect hosts seemed to be virtually null, as grasses infected with hybrid Epichloë were never found. This scenario suggests that E. clarkii and E. typhina have developed strong isolating barriers that maintain the species boundaries. However, the mechanisms that underlie the maintenance of the species barriers are largely unknown.

Addressing questions of speciation in sexual Epichloë species involves careful examination of the structure and hybridization of natural populations for which efficient molecular tools, such as microsatellites, are necessary. Previously, microsatellite markers have been developed to study the ecology and diversity of E. bromicola and Neotyphodium spp. [28, 29], and for identifying different sexual and asexual Epichloë endophytes in planta [30-32]. Most Neotyphodium species, today classified in the genus Epichlö [33], are thought to be derived from ancestral Epichloë species, often as a result of ancient hybridization events [34, 35]. However, the available microsatellites yielded unsatisfactory results when tested on our samples, probably because of partial primer mismatches among Epichloë species.

In this study, we aimed to investigate the genetic signature of species delimitation in the case of the two sympatric populations of E. typhina and E. clarkii at the Aubonne site in order to gain insight in the genetic differentiation of the fungal species and provide genetic evidence of their effective reproductive isolation. To achieve this goal, we developed a new set of microsatellite markers starting from whole genome sequences of Epichloë poae [36] to detect markers that are potentially applicable to a wide range of species in the genus. To our knowledge, this study is the first that addresses the genetics behind reproductive isolation among sympatric Epichloë species.

\section{Material and Methods}

\section{Computational Workflow for Microsatellite Design}

A workflow was developed to find, in silico test and filter microsatellites in the genomic DNA sequences of $E$. poae strain E5819 downloaded from Genome Projects at University of Kentucky (http://www.endophyte.uky.edu). The workflow employed in-house Perl scripts calling the Tandem Repeats Finder v. 4.0.4 [37] to find repeats, Primer3 v. 2.3.0 [38] to construct primers in the flanking regions, and BLAST+ v. 2.2. 26 [39] for in silico primer testing on the genome.

Primer pairs were designed in order to obtain PCR products between 100 and $500 \mathrm{bp}$ long. The workflow allowed for filtering of the microsatellites into PCR fragment size classes and motif length classes. Primers with more than one potential PCR product were discarded. Furthermore, the primers were in silico tested on the genome of the grass species 
Brachypodium distachyon strain Bd21-1 downloaded from the Brachypodium Genome Database (http://www. brachypodium.org) to reduce the potential risk of crossamplification on the genome of the host plants. This should allow better use of the primers for experiments targeting the endophytes in planta. An additional primer blasting against $E$. festucae strain E894 genome (Genome Projects at University of Kentucky) with up to two mismatches was performed to identify shared repeats with a closely related species and thus create a large dataset of markers potentially applicable to a broad taxonomic range within the genus.

\section{Primer Testing}

Lab protocols of newly developed microsatellites were initially tested on a set of samples, hereafter named "lab strains," which included pure cultures of E. poae, E. typhina, E. clarkii, and Epichloë bromicola and F1 progeny of four crosses (i.e., E. clarkii $\times$ E. clarkii, E. typhina $\times$ E. typhina, E. clarkii $\times$ E. typhina, and E. typhina $\times E$. clarkii; Table 1) grown on potato dextrose agar (PDA; BD Comp., Sparks, MD, USA) to test the detectability of hybrids, should they occur in natural populations. Crosses were performed in the greenhouse on freshly emerging stromata on the grasses from which the parental fungal strains were collected.

Microsatellite sequences were in silico screened for $100 \%$ perfect di-, tri-, tetra-, penta-, and hexanucleotides on the genome of $E$. poae with an optimal annealing temperature between 58 and $60^{\circ} \mathrm{C}$ [40]. In total, 75,432 potential primer pairs amplifying 8451 microsatellites were generated by our workflow from $E$. poae whole genome sequences. Out of these, 8136 primer pairs covered 1084 microsatellites with motifs repeated 5-20 times. Fifty-one primer pairs were randomly chosen across repeat types and labeled with cost-efficient M13 [41] to sort out non-working microsatellites in preliminary tests performed on the lab strains. The PCR volume of $10 \mu \mathrm{l}$ contained approximately $1 \mathrm{ng}$ of genomic DNA, with $5 \times$ PCR

Table 1 Species with their respective hosts and experimental hybrid progeny with numbers of isolates used in this study and the number of microsatellites out of 38 tested that successfully amplified with M13

\begin{tabular}{llll}
\hline Fungal species & Host & $\begin{array}{l}\text { No. of } \\
\text { isolates }\end{array}$ & $\begin{array}{l}\text { No. of } \\
\text { microsatellites }\end{array}$ \\
\hline E. poae & Poa nemoralis & 3 & 38 \\
E. typhina & Dactylis glomerata & 4 & 37 \\
E. clarkii & Holcus lanatus & 3 & 36 \\
E. bromicola & Bromus erectus & 2 & 31 \\
E. clarkii $\times$ E. clarkii & Progeny & 6 & 35 \\
E. typhina $\times$ E. typhina & Progeny & 4 & 38 \\
E. clarkii $\times$ E. typhina & Progeny & 4 & 37 \\
E. typhina $\times$ E. clarkii & Progeny & 4 & 37 \\
\hline
\end{tabular}

Buffer (Promega), $5 \mathrm{U} / \mu \mathrm{l}$ Go-Taq Polymerase (Promega, Madison, WI, USA), $25 \mathrm{mM} \mathrm{MgCl} 2,2.5 \mathrm{mM}$ of each dNTP, $2.5 \mu \mathrm{M}$ of forward primer, and $2.5 \mu \mathrm{M}$ of each reverse and universal FAM-labeled M13 primers. Amplification was carried out separately for each locus on Sensoquest labcycler (Witec AG, Luzern, Switzerland) with initial denaturing at $94{ }^{\circ} \mathrm{C}$ for $3 \mathrm{~min}$, followed by 30 cycles of $94{ }^{\circ} \mathrm{C}$ for $30 \mathrm{~s}$, $60^{\circ} \mathrm{C}$ for $30 \mathrm{~s}$, and $72{ }^{\circ} \mathrm{C}$ for $30 \mathrm{~s}$, followed by 8 cycles of $94^{\circ} \mathrm{C}$ for $30 \mathrm{~s}, 52^{\circ} \mathrm{C}$ for $30 \mathrm{~s}$, and $72{ }^{\circ} \mathrm{C}$ for $30 \mathrm{~s}$ with a final extension of $60{ }^{\circ} \mathrm{C}$ for $10 \mathrm{~min}$. All PCR products were run with GeneScan-500 LIZ as size standard on a 3130 Genetic Analyzer (Applied Biosystems, Foster City, CA, USA), and electropherograms were analyzed using Geneious 6.1.8 [42].

Out of 51 tested M13 primer pairs, 38 successfully amplifying were retained (Table 1). Sixteen of the microsatellites consisted of dinucleotide repeats, 12 of trinucleotide repeats, eight of tetranucleotide repeats, one of a pentanucleotide motif, and one of a hexanucleotide motif. Successful amplification of these primers across our lab strains ranged from 82 to $100 \%$ (Table 1). The targeted PCR fragments containing microsatellites averaged $294 \mathrm{bp}$ in length and ranged between 107 and $478 \mathrm{bp}$.

Eventually, 15 polymorphic markers were chosen based on clean and consistent peaks without stuttering in order to decrease the error rate. To create PCR multiplex sets, primer pairs were selected according to their different product sizes and labeled with one of three fluorescent labels (6-FAM, HEX, and ATTO0550). Finally, markers were arranged in four multiplex sets (Table 2) and tested on the lab strains using a PCR volume of $10 \mu \mathrm{l}$ containing approximately $1 \mathrm{ng}$ of genomic DNA, $2 \times$ Multiplex Mastermix (Multiplex PCR Kit, Quiagen), and $10 \times$ Primermix $(2 \mu \mathrm{M})$. Amplification was carried out on Sensoquest labcycler (Witec AG) with initial denaturing at $95{ }^{\circ} \mathrm{C}$ for $5 \mathrm{~min}$, followed by 28 cycles of $95^{\circ} \mathrm{C}$ for $30 \mathrm{~s}, 58{ }^{\circ} \mathrm{C}$ for $90 \mathrm{~s}$, and $72{ }^{\circ} \mathrm{C}$ for $30 \mathrm{~s}$, with a final extension of $60{ }^{\circ} \mathrm{C}$ for $30 \mathrm{~min}$.

\section{Genotyping of Natural Populations}

Sympatric host grasses infected by E. typhina and E. clarkii were sampled in Spring 2013 at a natural grassland site of approximately 2 ha in Aubonne, Canton Vaud, Switzerland. One stroma of each infected plant occurring along eight transects across the population were collected every $5 \mathrm{~m}$ resulting in 27 stromata of E. typhina and 50 of E. clarkii. Approximately $10 \%$ of the estimated number of individuals of E. typhina and $25 \%$ of those of E. clarkii were thus sampled. Additionally, in order to compare genetic distances between the two sympatric species to those of a third taxon, 22 samples of $E$. poae from three different locations were included in the analysis. The host of $E$. poae occurs in sympatry with E. typhina and E. clarkii at one edge of the population in Aubonne, but the rare occurrence of the fungus at this site allowed collecting only three samples. Eighteen additional 
Table 2 Repeat motifs, primer sequences, and size ranges of 15 selected microsatellite loci from Epichloë spp. used in four (M1-M4) fluorescent labeled multiplexes

\begin{tabular}{|c|c|c|c|c|c|}
\hline Mix & Locus & Dye & Repeat motif & Primer sequence $\left(5^{\prime}-3^{\prime}\right)$ & Size range (bp) \\
\hline \multirow[t]{8}{*}{ M1 } & \multirow[t]{2}{*}{ E29 } & \multirow[t]{2}{*}{$\mathrm{H}$} & \multirow[t]{2}{*}{$(\mathrm{AGC})_{9}$} & F: TTCCAGCAGCTCTTCAATACC & \multirow[t]{2}{*}{$129-201$} \\
\hline & & & & R: ACAGTGGTTCCTGAGGTTTGA & \\
\hline & \multirow[t]{2}{*}{ E8 } & \multirow[t]{2}{*}{$\mathrm{F}$} & \multirow[t]{2}{*}{$(\mathrm{AC})_{14}$} & F: CATGGACCAAGTTGTGAGACC & \multirow[t]{2}{*}{$226-266$} \\
\hline & & & & R: AGCAAGTCTCGTAACGGTCTG & \\
\hline & \multirow[t]{2}{*}{ E50 } & \multirow[t]{2}{*}{$\mathrm{H}$} & \multirow{2}{*}{$(\mathrm{TTG})_{12}$} & F: TCGTCTTGGACTTTGCCTTT & \multirow[t]{2}{*}{$315-378$} \\
\hline & & & & R: TTGAGGTTGTCGAGATACACG & \\
\hline & \multirow[t]{2}{*}{ E39 } & \multirow[t]{2}{*}{$\mathrm{F}$} & \multirow[t]{2}{*}{$(\text { GTTTC })_{12}$} & F: GTAGCACATGCATCGAATCAG & \multirow[t]{2}{*}{$425-500$} \\
\hline & & & & R: ACCCACTAAAGACGGATGACA & \\
\hline \multirow[t]{8}{*}{ M2 } & \multirow[t]{2}{*}{$\mathrm{E} 47$} & \multirow[t]{2}{*}{$\mathrm{H}$} & \multirow[t]{2}{*}{$(\mathrm{CTCA})_{9}$} & F: GCCTGTTGAGAAAGACGTGAT & \multirow[t]{2}{*}{$286-354$} \\
\hline & & & & R: GATCGAAACACGGGATCATAC & \\
\hline & \multirow[t]{2}{*}{ E32 } & \multirow[t]{2}{*}{ A } & \multirow[t]{2}{*}{$(\mathrm{CAG})_{11}$} & F: AGATGAATGGTCAGCAGTTCC & \multirow[t]{2}{*}{$326-347$} \\
\hline & & & & R: GGACCATACTTCGTCAACGTC & \\
\hline & \multirow[t]{2}{*}{ E45 } & \multirow[t]{2}{*}{ A } & \multirow[t]{2}{*}{$(\mathrm{GT})_{15}$} & F: TTGACGTCGGGAGGTAGTAGA & \multirow[t]{2}{*}{$376-466$} \\
\hline & & & & R: CTGGTTACGGAAAGCGAGATA & \\
\hline & \multirow[t]{2}{*}{ E13 } & $\mathrm{F}$ & $(\mathrm{GA})_{11}$ & F: GTTCTCCAAGGCTTCCAATTT & $485-551$ \\
\hline & & & & R: GAGAAACGATATTCGCATTGG & \\
\hline M3 & E4 & $\mathrm{F}$ & $(\mathrm{AG})_{9}$ & F: ATTGACCTGTAGCGCGAGTAG & $126-170$ \\
\hline & & & & R: CAGAACCAATTCGAATCCATC & \\
\hline & E33 & $\mathrm{F}$ & $(\mathrm{TCG})_{11}$ & F: TGCCAGATGTTTCAATGACTG & $329-338$ \\
\hline & & & & R: AACCCATACTCAGCTTTGCAG & \\
\hline & E36 & $\mathrm{H}$ & $(\mathrm{TGC})_{7}$ & F: ATTCGAGAATGGATGACCTGA & $393-417$ \\
\hline & & & & R: AAGAAAGGAATGGGATTGCTC & \\
\hline M4 & E22 & $\mathrm{F}$ & $(\text { TGGA })_{10}$ & F: GCAAGGATTGGTTGGTGATAA & $124-152$ \\
\hline & & & & R: GCGGATCACTCTGTAGGCTAA & \\
\hline & $\mathrm{E} 27$ & $\mathrm{H}$ & $(\mathrm{GA})_{8}$ & F: TATAAATGACGCTGGGCTTGT & $370-428$ \\
\hline & & & & R: TGCACTTGAAGAAGCCATGTA & \\
\hline & E11 & $\mathrm{F}$ & $(\mathrm{CT})_{11}$ & F: GTCAGAGGGCAGTAGTGACG & $265-289$ \\
\hline & & & & R: ATGTAATGCTCTGCCTGCTTC & \\
\hline & E46 & A & $(\mathrm{AG})_{9}$ & F: TCGTGACACCTTCTTCGGTAT & $382-418$ \\
\hline & & & & R: AGAGGTTGTCGTGAGCATCAT & \\
\hline
\end{tabular}

$H$ Hex, $F$ 6-Fam, $A$ ATTO0550

samples came from the Botanical Garden in Zurich and one from Zollikerberg in Zurich. DNA was extracted from cultured fungi and, in particular, from each stroma using mycelium from the interior part of the stroma split open under sterile conditions. DNA extraction followed the cetyltrimethyl ammonium bromide (CTAB) standard protocol [43]. Extracted DNA was subjected to electrophoresis to confirm the presence of template for PCR and quantified using a Nanodrop spectrophotometer (Thermo Scientific, Inc., Waltham, MA, USA).

The 15 selected markers in four multiplexes described above were used to study the population structure of E. typhina, E. clarkii, and E. poae at the Aubonne site. In order to test whether genetic diversity in the three species was also detectable in gene sequences, a portion of tef $A$ nuclear region was sequenced for ten, 15 , and seven representative samples of E. typhina, E. clarkii, and E. poae, respectively. The tefA gene is commonly used for distinguishing described sexual Epichloë species, as it is rich in polymorphic introns allowing for sufficient resolution [22]. PCR reactions were performed using primers 5'-GGG TAA GGA CGA AAA GAC TCA-3' (forward) and 5'-CGG CAG CGA TAA TCA
GGA TAG-3' (reverse) for tefA as previously described [44], and sequenced using BigDye Terminator v. 3.1 on a 3130 Genetic Analyzer (Applied Biosystems, Foster City, CA, USA). Forward and reverse strands were assembled in Geneious v. 6.1.8 [42], and consensus sequences were aligned using the default plugin of the software. The obtained nucleotide sequences have been submitted to GenBank under accessions KP064360-KP064391.

Data Analysis of Natural Populations

Number of alleles, allelic richness at each locus, gene diversity, as well as genotypic linkage disequilibrium based on 10 , 500 permutations were determined using the program FSTAT v. 2.9 .3 [45]. In the analyses of linkage disequilibrium, the data were coded as diploid data, where single-allelic loci were considered to be homozygous. The genetic differentiation among species was assessed in POPGENE v. 1.31 [46] using Fisher's exact tests and pairwise $F_{\text {ST }}$ statistics [47]. The software program GENALEX v. 6.2 [48] was used to analyze the molecular variance (AMOVA) among and within species of E. typhina, E. clarkii, and E. poae with 9999 permutations. 
Sequential Bonferroni corrections were performed for simultaneous multiple tests [49].

To infer population structure of E. typhina, E. clarkii, and E. poae, $\mathrm{PCO}-\mathrm{MC}$ analysis was used, a method that combines principal coordinate analysis (PCO) with testing of significant clusters for population structure [50]. Following the authors' recommendations (http://lamar.colostate.edu/ reevesp/ PCOMC/PCOMC.html), stability of clusters was first assessed with a cut-off set to 15 , and significance was then tested at the 0.05 level. Furthermore, the Bayesian modelclustering algorithm implemented in the program STRU CTURE v. 2.3.2 [51, 52] was also employed. Iteration parameters were set to 950,000 Monte Carlo Markov Chain (MCMC) iterations preceded by a burn-in period of 50,000 iterations. Ten independent simulations were performed to test for the consistency of the results for $K$ ranging from 1 to 5 . To infer the number of clusters, i.e., $K$, that best fit the data the method described by Evanno et al. [53] as implemented in STRUCTURE HARVESTER was used [54]. Membership coefficients estimated by STRUCTURE were visualized using the software programme DISTRUCT v. 1.1 [55].

\section{Results}

\section{Primer Design}

Blasting of the total 75,432 primer pairs generated in this study against Epichloe festucae revealed that 10,406 primer pairs covering 2022 microsatellites with $100 \%$ perfectness are potentially suitable also for application on other Epichloë species. By restricting the repeat number of the microsatellite motifs to $5-20,885$ primer pairs covering 180 microsatellites remained. The full list of these candidate loci with respective descriptions and primer combinations is available as supplementary material (Tab. S1). Within the full set of microsatellites, the motif of hexanucleotide repeats $(67 \%)$ was the most abundant class, followed by trinucleotide $(15 \%)$, pentanucleotide $(12 \%)$, tetranucleotide $(5 \%)$, and dinucleotide repeats (1\%; Fig. 1a). In contrast, when filtering for 5-20 motif repeats, trinucleotide repeats (79 \%) were the most abundant, followed by dinucleotide $(15 \%)$, tetranucleotide $(3 \%)$, pentanucleotide $(2 \%)$, and hexanucleotide repeats ( $1 \%$; Fig. 1b). Among the 15 fluorescent labeled primers developed on E. poae, seven were recovered also in the genome of $E$. festucae and yielded successful PCR products when tested in vitro. Of the remaining eight microsatellites, three yielded successful PCR products in E. festucae, despite the fact that they were not found in silico in this species. This indicates that microsatellites can be missed if a draft version of the genome is used and that unsuccessful blasting does not necessarily translate into negative in vitro results.

Genetic Diversity and Structure of Sympatric Epichloë Species

The allelic diversity of 99 isolates of E. typhina, E. clarkii, and $E$. poae was analyzed within each species at 15 polymorphic loci. The number of alleles per locus ranged from 2 to 12 for E. typhina, from 1 to 14 for E. clarkii and from 1 to 4 for E. poae. The allelic richness corrected for uneven sample size ranged from 2 to 11.87 per locus for E. typhina, from 1 to 12.86 for E. clarkii, and from 1 to 4 for E. poae (Table 3). The mean allelic richness across the three species was 9.58 , of which 6.07 alleles were observed across isolates from E. typhina, 6.55 alleles in E. clarkii, and 2.33 alleles were observed among isolates from E. poae. All loci from E. typhina were polymorphic, whereas E. clarkii and E. poae had 93 and $80 \%$ polymorphic loci, respectively. Overall, $91 \%$ of the loci from the three species were polymorphic. Significant linkage disequilibrium $\left(P_{\text {adjust }}<0.0005\right)$ was detected in one, two and four of the 105 possible pairwise combinations of loci in E. typhina, E. clarkii, and E. poae, respectively. The mean total gene diversity $(H)$ calculated using the allele frequencies across all isolates was 0.53 . This was similar to the values obtained for gene diversity estimates, namely, 0.664 and 0.628 , in isolates from E. typhina and E. clarkii, respectively, and 0.345 observed in isolates from E. poae (Table 3).
Fig. 1 Frequencies of different classes of nucleotide repeats for microsatellites compatible in E. poae and E. festucae. a Microsatellites (2022) with any number of motif repeat. $\mathbf{b}$ Microsatellites (180) with motifs repeated 5-20 times
A

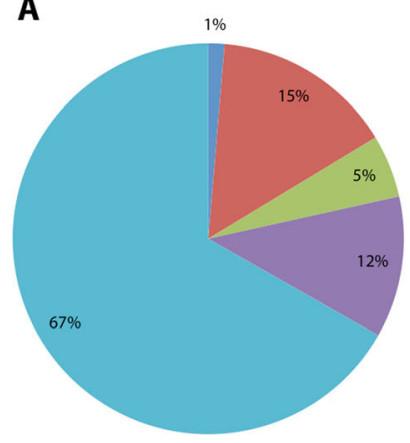

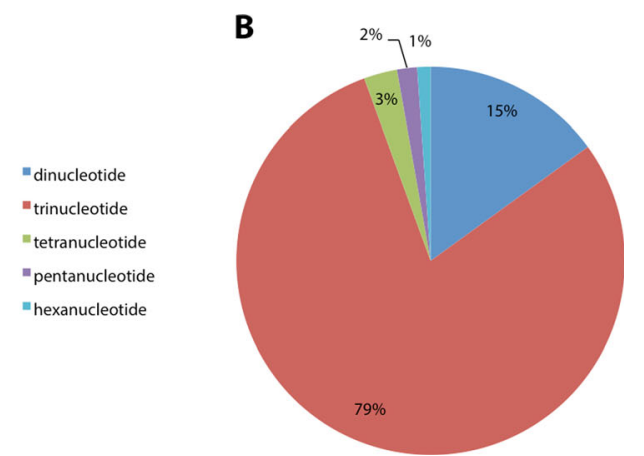


Table 3 Number of alleles, allelic richness, and gene diversity per locus at 15 microsatellite loci for E. typhina, E. clarkii, and E. poae

\begin{tabular}{|c|c|c|c|c|c|c|c|c|c|}
\hline \multirow[t]{2}{*}{ Locus } & \multicolumn{3}{|c|}{ E. typhina } & \multicolumn{3}{|c|}{ E. clarkii } & \multicolumn{3}{|c|}{ E. poae } \\
\hline & $\mathrm{Na}$ & $\mathrm{R}$ & $\mathrm{H}$ & $\mathrm{Na}$ & $\mathrm{R}$ & $\mathrm{H}$ & $\mathrm{Na}$ & $\mathrm{R}$ & $\mathrm{H}$ \\
\hline E8 & 5 & 4.937 & 0.709 & 7 & 6.346 & 0.711 & 3 & 3 & 0.325 \\
\hline E39 & 2 & 2.000 & 0.262 & 8 & 6.938 & 0.712 & 2 & 2 & 0.455 \\
\hline E29 & 10 & 9.872 & 0.875 & 14 & 12.185 & 0.885 & 4 & 4 & 0.571 \\
\hline E50 & 9 & 8.904 & 0.855 & 9 & 7.751 & 0.820 & 2 & 2 & 0.247 \\
\hline E13 & 12 & 11.871 & 0.909 & 11 & 10.225 & 0.796 & 2 & 2 & 0.485 \\
\hline $\mathrm{E} 47$ & 6 & 5.968 & 0.672 & 8 & 7.368 & 0.839 & 2 & 2 & 0.519 \\
\hline E32 & 5 & 4.999 & 0.658 & 4 & 3.370 & 0.223 & 2 & 2 & 0.247 \\
\hline $\mathrm{E} 45$ & 10 & 9.873 & 0.892 & 10 & 8.566 & 0.770 & 4 & 4 & 0.519 \\
\hline $\mathrm{E} 4$ & 3 & 2.969 & 0.544 & 8 & 6.946 & 0.758 & 3 & 3 & 0.606 \\
\hline E33 & 2 & 2.000 & 0.359 & 4 & 3.906 & 0.643 & 1 & 1 & 0.000 \\
\hline E36 & 6 & 5.937 & 0.744 & 2 & 1.689 & 0.040 & 2 & 2 & 0.247 \\
\hline E22 & 2 & 2.000 & 0.484 & 6 & 5.190 & 0.618 & 3 & 3 & 0.394 \\
\hline E11 & 5 & 4.936 & 0.567 & 1 & 1.000 & 0.000 & 1 & 1 & 0.000 \\
\hline E27 & 12 & 11.840 & 0.892 & 11 & 9.797 & 0.817 & 1 & 1 & 0.000 \\
\hline E46 & 3 & 2.969 & 0.544 & 8 & 7.032 & 0.783 & 3 & 3 & 0.558 \\
\hline Mean & 6 & 6.072 & 0.664 & 7 & 6.554 & 0.628 & 2 & 2.333 & 0.345 \\
\hline
\end{tabular}

$N a$ number of alleles per locus, $R$ corrected allelic richness, $H$ gene diversity

The observed levels of genetic differentiation (Fisher's exact test $P<0.001$ ) among E. typhina, E. clarkii, and $E$. poae reflect a clear structuring of the three species. These results were supported by high pairwise $F_{\mathrm{ST}}$ values of 0.446 between E. typhina and E. poae, 0.453 between E. clarkii and $E$. poae, and 0.280 between the more closely related E. typhina and E. clarkii (Table 4). The AMOVA confirmed these results by showing that $62 \%$ of the molecular variance is found within species and $38 \%$ among species (Table 5).

PCO-MC analyses revealed three stable and statistically significant clusters $(P<0.05)$ that corresponded to the three host-specific species (Fig. 2). In this analysis, $19 \%$ of the variation was explained by the first axis, $11.6 \%$ by the second axis, and $4.3 \%$ by the third axis. No samples were found in between the clusters indicating that all samples are assigned to the three genotypically separate species. The STRUCTURE analysis yielded results consistent with those obtained from PCO-MC. The distribution of the maximum likelihood was the highest for $K=3$. The first cluster included all genotypes of

Table 4 Pairwise $F_{\mathrm{ST}}$ values among E. typhina, E. clarkii, and E. poae

\begin{tabular}{lll} 
& E.typhina & E.clarkii \\
\hline E. clarkii & $0.280^{*}$ & \\
E. poae & $0.446^{*}$ & $0.453^{*}$ \\
\hline
\end{tabular}

An asterisk indicated the $F_{\mathrm{ST}}$ value is highly significantly different from 0 $(P<0.001) . P$ values were adjusted using the Bonferroni procedure
Table 5 AMOVA results testing the genetic structure among E. typhina, E. clarkii, and E. poae at 15 microsatellite loci (9999 permutations)

\begin{tabular}{lrrlll}
\hline $\begin{array}{l}\text { Source of } \\
\text { variation }\end{array}$ & $d f$ & $\begin{array}{l}\text { Sum of } \\
\text { squares }\end{array}$ & $\begin{array}{l}\text { Variance } \\
\text { components }\end{array}$ & $\begin{array}{l}\text { Percentage } \\
\text { of variation }\end{array}$ & P value \\
\hline Among species & 2 & 172.57 & 2.67 & 38 & $<0.001$ \\
Within species & 96 & 414.53 & 4.32 & 62 & \\
Total & 98 & 587.10 & 6.98 & & \\
\hline
\end{tabular}

isolates representing E. typhina, while the second cluster consisted of genotypes of $E$. clarkii and the third cluster of E. poae. The assignment of the individuals in the plot showed very reduced levels of admixture.

The alignment of the sequences of the nuclear gene tef $A$ was $\sim 620$ bp in length and showed a considerable number of species-specific fixed single nucleotide polymorphisms (SNPs) for all three species of the study. In particular, four, three, and eight SNPs were found to be fixed among representatives of E. typhina, E. clarkii, and E. poae, respectively. Hence, these SNPs have diagnostic value for the identification of E. typhina, E. clarkii, and E. poae (Fig. S1).

\section{Discussion}

Genetic Diversity and Structure of Sympatric Epichloë Species

Host specificity is hypothesized to promote reproductive isolation between sympatric fungal species when mating is constrained to occur within the host [1]. Evidence of the role of host specificity in maintaining species boundaries has emerged from a number of systems including Ascochyta species, Mycosphaerella graminicola, and Microbotryum species; hence, this mechanism is hypothesized to play an important role in the speciation of these fungi $[12,13,56]$. To date, no in-depth study has addressed the validity of species boundaries in host-specific endophytes, and we contribute to fill this gap by presenting evidence from sympatric populations of Epichloë.

Our study provides clear evidence of genetic discontinuity between E. typhina and E. clarkii, indicating that reproductive isolation mechanisms exist to keep sympatric populations both morphologically and genetically distinct. Moreover, although the third species (E. poae) was represented by only three samples from Aubonne, these had clearly distinct genotypes that clustered with additional samples of this species from two different geographical regions. This offers preliminary evidence that also $E$. poae is maintained isolated from E. typhina and E. clarkii. In fact, both PCO-MC and Bayesian 
A

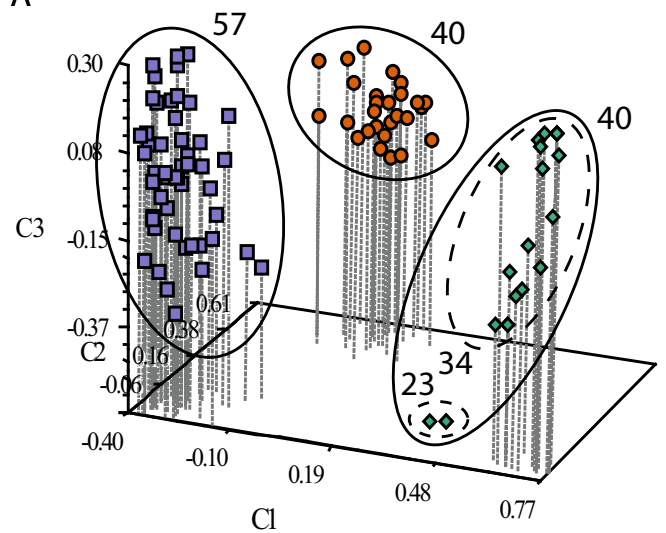

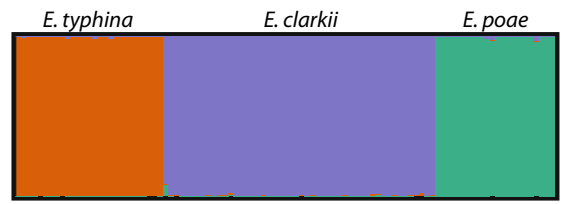

Fig. 2 a PCO-MC analysis based on 15 microsatellite loci for E. typhina (circles), E. clarkii (squares), and E. poae (diamonds). Solid lines indicate stable and statistically significant $(P<0.05)$ clusters, whereas dashed line indicate stable clusters that do not pass the significance threshold. b
Bayesian clustering of E. typhina, E. clarkii, and E. poae at 15 microsatellite loci as inferred from STRUCTURE under the assumption of $K=3$ populations

the genetic structure of populations from asexually reproducing Epichloë species, to our knowledge no study has addressed the genotypic delimitation of sympatric sexually reproducing taxa.

This study showed high genetic differentiation among populations with pairwise $F_{\mathrm{ST}}$ values of 0.446 between $E$. typhina and E. poae, 0.453 between E. clarkii and E. poae, and 0.280 between E. typhina and E. clarkii. The higher $F_{\mathrm{ST}}$ value of $E$. poae in pairwise comparisons with the other two species indicates that E. poae is a more genetically isolated taxon, supporting previous results [22]. While this result can be caused by the geographic isolation of a great proportion of the E. poae samples external to the Aubonne site, comparisons of the population statistics among the three species reveal peculiar characteristics for this taxon. We found similar levels of allelic richness for E. typhina and E. clarkii, but these values were more than two times higher than for $E$. poae (Table 3). Furthermore, E. poae showed lower percentage of polymorphic loci and lowest gene diversity (Table 3), despite the fact that it was represented by samples from three different locations. These characteristics of $E$. poae may be explained by its different transmission mode. In contrast to E. typhina and $E$. clarkii that are only horizontally transmitted by sexual ascospores, E. poae can transmit both horizontally and vertically by seed. This may lead more frequently to clonal endophytes in plants growing from seeds. Accordingly, only 16 genotypes out of 22 were unique in E. poae, whereas in E. typhina and E. clarkii all genotypes were found to be unique.

\section{Reproductive Isolation of Epichloë Species}

Hypotheses on the origin of reproductive barriers should include both intrinsic and extrinsic mechanisms, and our results provide preliminary insights to evaluate alternative ated subpopulations [61]. While evidence is accumulating on 
scenarios. An obvious mechanism of extrinsic prezygotic isolation includes the preferential transfer of spermatia by Botanophila flies that actively contribute to exchange of gametes. Yet, given that Botanophila flies are largely unspecific in their visiting behavior, spermatia from different hosts are collected and actively transported in mixtures among the stromata [27]; thus, a preference by fly vectors is unlikely to constitute an efficient barrier. Alternatively, gene flow between Epichloë species could be restricted via prezygotic assortative mating on the stromata, if individuals were able to discriminate between conspecific and heterospecific spores. Preliminary data in E. typhina and E. clarkii showed that the outcome of matings depends on the concentrations of conspecific and heterospecific spores applied in mixtures on the stroma (Schirrmann, unpublished data). For example, if conspecific and heterospecific spores were applied in equal concentrations, only progeny of intraspecific matings were found, thus indicating a preference for spores from the same species. These results are consistent with previous evidence for effective interference between matings of different Epichloë species on different parts of the stroma surface, depending upon the fungal strains involved [62]. The observed interferences suggested that signals generated after positive mating could be transported throughout the stroma and thus prevent or abort other mating interactions on the same stroma.

In contrast, intrinsic postzygotic isolation is often associated with hybrid sterility or inviability as shown by in vitro crosses that are initiated and then later aborted [2]. In Neurospora species, interspecific compared to intraspecific crosses resulted in fewer viable ascospores, and Microbotryum species produced fewer viable mycelia in interspecific crosses [9, 63]. Furthermore, in Saccharomyces species, the incompatibility between genes of the nuclear and mitochondrial genomes causes hybrid sterility, which might have evolved as a by-product of ecological adaptation to different nutrient sources [64]. In our system, the viability of experimentally generated hybrids between species of the E. typhina complex was not reduced, and their radial growth rates in vitro were not significantly different, suggesting a lack of obvious intrinsic postzygotic isolation barriers between the parental strains (Schirrmann, unpublished data). Therefore, postzygotic barriers may depend on extrinsic factors as for example a decreased fitness of the hybrids due to a likely maladaptation to host grasses, preventing infection or persistence of hybrids. Host specificity was shown in a study by Chung et al. [65], where the seedling infection frequency and stability of E. typhina isolates and their progeny were tested. A reduced infectivity of interspecific progeny in parental hosts was demonstrated previously also for E. typhina and E. clarkii [66] and was confirmed by reciprocal inoculation experiments for these species and E. poae (Schirrmann, unpublished data).

Overall, the evidence collected so far indicates that natural populations of sympatric Epichloë species may be genetically differentiated due to assortative mating on the stroma and host specificity of parental strains. In the future, further investigations will hopefully test these hypotheses thoroughly.

\section{Primer Design and Testing}

In this study, we developed new microsatellite markers to study population structure at the interspecific level within the E. typhina complex. Of the 38 successfully amplifying microsatellites tested on the investigated lab strains, i.e., E. typhina, E. clarkii, E. poae, and $E$. bromicola, and the four progeny from inter- and intraspecific crosses, $82-100 \%$ yielded a PCR product. This suggests that our set of loci has a high probability of cross-species transferability within Epichloë and that hybrids could be detected if they would be present in nature (Table 1). Additionally, the large set of markers identified in $E$. poae and successfully in silico verified in E. festucae provides a valuable resource of candidate primers applicable to a broad taxonomic range of Epichloë. However, careful testing should always be performed to optimize amplification protocols and multiplex design, as well as to rule out unspecific amplification products. Overall, the loci detected and tested here constitute a substantial genetic resource that complements previous microsatellite markers developed for Epichloë/Neotyphodium species [28-31].

\section{Conclusions}

Using a new set of 15 microsatellites, we assessed the population structure of sympatric species of the E. typhina complex, i.e., E. typhina, E. clarkii, and E. poae, at a natural field site in Aubonne, Switzerland. Our results showed that high genetic differentiation and very limited gene flow exist among these three species, indicating that mechanisms of reproductive isolation keep natural Epichloë populations genetically distinct. Preliminary evidence suggests that host specificity and maladaptation of hybrids to host grasses may act as reproductive isolation barriers in Epichloë and therefore promote their speciation. However, further studies are needed to disentangle the complex interplay of isolation barriers that may be responsible to maintain species boundaries in natural populations.

Acknowledgments This study was funded by the Swiss National Science Foundation (31003A_138479). Microsatellite data were generated in the Genetic Diversity Centre of ETH Zurich (GDC). C. L. Schardl gave advice and provided access to unpublished genome data. We thank B. Blattmann for laboratory assistance and M. C. Fischer and N. Zemp for providing methodological details. 


\section{References}

1. Kohn LM (2005) Mechanisms of fungal speciation. Annu Rev Phytopathol 43:279-308. doi:10.1146/annurev.phyto.43.040204. 135958

2. Giraud T, Refrégier G, Le Gac M et al (2008) Speciation in fungi. Fungal Genet Biol 45:791-802. doi:10.1016/j.fgb.2008.02.001

3. Restrepo S, Tabima JF, Mideros MF et al (2014) Speciation in fungal and oomycete plant pathogens. Annu Rev Phytopathol 52:289-316. doi:10.1146/annurev-phyto-102313-050056

4. Finlay BJ (2002) Global dispersal of free-living microbial eukaryote species. Science 296:1061-1063. doi:10.1126/science.1070710

5. Slatkin M (1987) Gene flow and the geographic structure of natural populations. Science 236:787-792

6. Liti G, Carter DM, Moses AM et al (2009) Population genomics of domestic and wild yeasts. Nature 458:337-341. doi:10.1038/ nature 07743

7. Ellison CE, Hall C, Kowbel D et al (2011) Population genomics and local adaptation in wild isolates of a model microbial eukaryote. Proc Natl Acad Sci U S A 108:2831-2836. doi:10.1073/pnas.1014971108

8. Koufopanou V, Burt A, Taylor JW (1997) Concordance of gene genealogies reveals reproductive isolation in the pathogenic fungus Coccidioides immitis. Proc Natl Acad Sci U S A 94:5478-5482

9. Dettman JR, Jacobson DJ, Turner E et al (2003) Reproductive isolation and phylogenetic divergence in Neurospora: comparing methods of species recognition in a model eukaryote. Evolution 57: 2721-2741

10. O'Donnell K, Ward TJ, Geiser DM et al (2004) Genealogical concordance between the mating type locus and seven other nuclear genes supports formal recognition of nine phylogenetically distinct species within the Fusarium graminearum clade. Fungal Genet Biol 41:600-623. doi:10.1016/j.fgb.2004.03.003

11. Fournier E, Giraud T (2008) Sympatric genetic differentiation of a generalist pathogenic fungus, Botrytis cinerea, on two different host plants, grapevine and bramble. J Evol Biol 21:122-132. doi:10.1111/ j.1420-9101.2007.01462.x

12. Peever TL (2007) Role of host specificity in the speciation of Ascochyta pathogens of cool season food legumes. Eur J Plant Pathol 119:119-126. doi:10.1007/s10658-007-9148-2

13. Stukenbrock EH, Bataillon T, Dutheil JY et al (2011) The making of a new pathogen: insights from comparative population genomics of the domesticated wheat pathogen Mycosphaerella graminicola and its wild sister species. Genome Res 21:2157-2166. doi:10.1101/gr. 118851.110

14. Giraud T (2006) Speciation in parasites: host switching does not automatically lead to allopatry. Trends Parasitol 22:151-152

15. Leuchtmann A (1992) Systematics, distribution, and host specificity of grass endophytes. Nat Toxins 1:150-162

16. Schardl CL, Scott B, Florea S, Zhang D (2009) Epichloë endophytes: clavicipitaceous symbionts of grasses. In: Deising HB (ed) The Mycota, vol V, Plant relationships, 2nd edition. Springer, Berlin, pp 275-306

17. Bultman TL, Leuchtmann A (2009) Biology of the Epichlö̈Botanophila interaction: an intriguing association between fungi and insects. Fungal Biol Rev 22:131-138. doi:10.1016/j.fbr.2009. 04.003

18. Scott B (2001) Epichloë endophytes: fungal symbionts of grasses. Curr Opin Microbiol 4:393-398

19. Western JH, Cavett JJ (1959) The choke disease of cocksfoot (Dactylis glomerata) caused by Epichloë typhina (Fr.) Tul. Trans Brit Mycol Soc 42:298-307

20. White JF Jr, Bultman TL (1987) Endophyte-host associations in forage grasses. VIII. Heterothallism in Epichloë typhina. Am J Bot $74: 1716-1721$
21. Bultman TL, White JF Jr, Bowdish TI, Welch AM (1998) A new kind of mutualism between fungi and insects. Mycol Res 102:235-238

22. Craven KD, Hsiau P, Leuchtmann A et al (2001) Multigene phylogeny of Epichloë species, fungal symbionts of grasses. Ann Missouri Bot Gard 88:14-34

23. Schardl CL, Leuchtmann A, Chung KR et al (1997) Coevolution by common descent of fungal symbionts (Epichloë spp.) and grass hosts. Mol Biol Evol 14:133-143

24. White JF Jr (1993) Endophyte-host associations in grasses. XIX A systematic study of some sympatric species of Epichloë in England. Mycologia 85:444 455

25. Leuchtmann A, Schardl CL (1998) Mating compatibility and phylogenetic relationships among two new species of Epichloë and other congeneric European species. Mycol Res 102:1169-1182

26. Leuchtmann A, Clay K (1997) The population biology of grass endophytes. In: Carroll GC, Tudzynski P (eds) The Mycota, vol V, Part B: Plant relationships. Springer-Verlag, Berlin, pp 185-202

27. Bultman TL, Leuchtmann A, Sullivan TJ, Dreyer AP (2011) Do Botanophila flies provide reproductive isolation between two species of Epichloë fungi? A field test. New Phytol 190:206-212. doi:10. 1111/j.1469-8137.2010.03612.x

28. Groppe K, Sanders I, Wiemken A, Boller T (1995) A microsatellite marker for studying the ecology and diversity of fungal endophytes (Epichloë spp.) in grasses. Appl Environ Microbiol 61:3943-3949

29. van Zijll de Jong E, Guthridge KM, Spangenberg GC, Forster JW (2003) Development and characterization of EST-derived simple sequence repeat (SSR) markers for pasture grass endophytes. Genome 46:277-290. doi:10.1139/g03-001

30. Groppe K, Boller T (1997) PCR assay based on a microsatellitecontaining locus for detection and quantification of Epichloë endophytes in grass tissue. Appl Environ Microbiol 63:1543-1550

31. Moon CD, Tapper BA, Scott B (1999) Identification of Epichloë endophytes in planta by a microsatellite-based PCR fingerprinting assay with automated analysis. Appl Environ Microbiol 65:12681279

32. Simpson MC, Wilken PM, Coetzee MPA et al (2013) Analysis of microsatellite markers in the genome of the plant pathogen Ceratocystis fimbriata. Fungal Biol 117:545-555. doi:10.1016/j. funbio.2013.06.004

33. Leuchtmann A, Bacon CW, Schardl CL et al (2014) Nomenclatural realignment of Neotyphodium species with genus Epichloë. Mycologia 106:202-215. doi:10.3852/13-251

34. Schardl CL, Craven KD (2003) Interspecific hybridization in plantassociated fungi and oomycetes: a review. Mol Ecol 12:2861-2873. doi:10.1046/j.1365-294X.2003.01965.x

35. Moon CD, Craven KD, Leuchtmann A et al (2004) Prevalence of interspecific hybrids amongst asexual fungal endophytes of grasses. Mol Ecol 13:1455-1467. doi:10.1111/j.1365-294X.2004.02138.x

36. Schardl CL, Young CA, Hesse U et al (2013) Plant-symbiotic fungi as chemical engineers: multi-genome analysis of the Clavicipitaceae reveals dynamics of alkaloid loci. PLoS Genet 9:e1003323. doi:10. 1371/journal.pgen.1003323.s012

37. Benson G (1999) Tandem repeats finder: a program to analyze DNA sequences. Nucleic Acids Res 27:573-580. doi:10.1093/nar/27.2. 573

38. Rozen S, Skaletsky JH (2000) Primer3 on the WWW for general users and for biologist programmers. In: Krawetz S, Misener S (eds) Bioinformatics methods and protocols: methods in molecular biology. Human Press, New York, pp 365-386

39. Camacho C, Coulouris G, Avagyan V et al (2009) BLAST+: architecture and applications. BMC Bioinformatics 10:421. doi:10.1186/ 1471-2105-10-421

40. Gardner MG, Fitch AJ, Bertozzi T, Lowe AJ (2011) Rise of the machines - recommendations for ecologists when using next generation sequencing for microsatellite development. Mol Ecol Resour 11:1093-1101. doi:10.1111/j.1755-0998.2011.03037.x 
41. Schuelke M (2000) An economic method for the fluorescent labeling of PCR fragments. Nat Biotechnol 18:233-234. doi:10.1038/72708

42. Drummond AJ, Ashton B, Buxton S, et al. (2013) Geneious v6.1 created by Biomatters. www.geneious.com

43. Doyle J, Doyle J (1987) A rapid DNA isolation procedure for small amounts of fresh leaf tissue. Phytochem Bull 19:11-15

44. Oberhofer M, Leuchtmann A (2012) Genetic diversity in epichloid endophytes of Hordelymus europaeus suggests repeated host jumps and interspecific hybridizations. Mol Ecol 21:2713-2726. doi:10. 1111/j.1365-294X.2012.05459.x

45. Goudet J (1995) FSTAT (version 1.2): a computer program to calculate F-statistics. J Hered 86:485-486

46. Yeh FC, Yang R-C, Boyle T (1999) POPGENE. Version 1.31. Microsoft Windows based freeware for population genetic analysis. In: University of Alberta, Alberta. Accessed 20 Feb 2014

47. Weir BS, Cockerham CC (1984) Estimating F-statistics for the analysis of population structure. Evolution 38:1358-1370

48. Peakall R, Smouse PE (2006) Genalex 6: genetic analysis in Excel. Population genetic software for teaching and research. Mol Ecol Notes 6:288-295. doi:10.1111/j.1471-8286.2005.01155.x

49. Rice AM (1989) Analyzing tables of statistical tests. Evolution 43: 223-225

50. Reeves PA, Richards CM (2009) Accurate inference of subtle population structure (and other genetic discontinuities) using principal coordinates. PLoS One 4:e4269. doi:10.1371/journal.pone. 0004269.s003

51. Pritchard JK, Stephens M, Donnelly P (2000) Inference of population structure using multilocus genotype data. Genetics 155:945-959

52. Falush D, Stephens M, Pritchard JK (2003) Inference of population structure using multilocus genotype data: linked loci and correlated allele frequencies. Genetics 164:1567-1587

53. Evanno G, Regnaut S, Goudet J (2005) Detecting the number of clusters of individuals using the software STRUCTURE: a simulation study. Mol Ecol 14:2611-2620. doi:10.1111/j.1365-294X.2005. 02553.x

54. Earl DA, vonHoldt BM (2012) STRUCTURE HARVESTER: a website and program for visualizing STRUCTURE output and implementing the Evanno method. Conserv Genet Res 4:359-361. doi:10.1007/s12686-011-9548-7

55. Rosenberg NA (2004) DISTRUCT: a program for the graphical display of population structure. Mol Ecol Notes 4:137-138. doi:10. 1046/j.1471-8286.2003.00566.x
56. Büker B, Petit E, Begerow D, Hood ME (2013) Experimental hybridization and backcrossing reveal forces of reproductive isolation in Microbotryum. BMC Evol Biol 13:1-1. doi:10. 1186/1471-2148-13-224

57. Sullivan TJ, Faeth SH (2004) Gene flow in the endophyte Neotyphodium and implications for coevolution with Festuca arizonica. Mol Ecol 13:649-656

58. Arroyo García R, Martínez Zapater JM, García Criado B, Zabalgogeazcoa Í (2002) Genetic structure of natural populations of the grass endophyte Epichloëfestucae in semiarid grasslands. Mol Ecol 11:355-364

59. Zhang X, Ren A, Ci H, Gao Y (2010) Genetic diversity and structure of Neotyphodium species and their host Achnatherum sibiricum in a natural grass-endophyte system. Microb Ecol 59:744-756. doi:10. 1007/s00248-010-9652-3

60. Wäli PR, Ahlholm JU, Helander M, Saikkonen K (2007) Occurrence and genetic structure of the systemic grass endophyte Epichloë festucae in fine fescue populations. Microb Ecol 53:20-29. doi:10. 1007/s00248-006-9076-2

61. Bucheli E, Leuchtmann A (1996) Evidence for genetic differentiation between choke-inducing and asymptomatic strains of the Epichloë grass endophyte from Brachypodium sylvaticum. Evolution 50: 1879-1887

62. Chung KR, Schardl CL (1997) Sexual cycle and horizontal transmission of the grass symbiont, Epichloë typhina. Mycol Res 101:295301. doi:10.1017/S0953756296002602

63. Le Gac M, Hood ME, Giraud T (2007) Evolution of reproductive isolation within a parasitic fungal species complex. Evolution 61: 1781-1787. doi:10.1111/j.1558-5646.2007.00144.x

64. Lee H-Y, Chou J-Y, Cheong L et al (2008) Incompatibility of nuclear and mitochondrial genomes causes hybrid sterility between two yeast species. Cell 135:1065-1073. doi:10.1016/ j.cell.2008.10.047

65. Chung KR, Hollin W, Siegel MR, Schardl CL (1997) Genetics of host specificity in Epichloë typhina. Phytopathology 87:599-605. doi:10.1094/PHYTO.1997.87.6.599

66. Leuchtmann A, Steinebrunner F (2012) Evidence for reproductive isolation in Epichloë - a case study. In: Young CA, Aiken GE, McCulley RL, Strickland JR (eds) Epichloae, endophytes of cool season grasses: implications, utilization and biology. The Samuel Roberts Noble Foundation, Ardmore, pp 113-116 